\title{
METACOGNICIÓN Y COMPRENSIÓN LECTORA CON TEXTOS BILINGES
}

\author{
WILMER RIVERA FUENTES ${ }^{1}$ \\ ${ }^{1}$ Universidad Nacional De San Cristóbal De Huamanga. Ayacucho, Perú.
}

\section{RESUMEN}

La investigación tiene como propósito explicar la relación entre las estrategias metacognitivas y la comprensión lectora en estudiantes de la serie 200 de la EFP de Enfermería UNSCH, 2019. El contexto sociolingüístico de los estudiantes es principalmente bilingüe quechua-castellano. La gran parte de los participantes de la muestra están clasificados de acuerdo al grado de dominio de las lenguas en los distinto niveles de bilingüismo, entre ellos: incipiente, subordinando y coordinado (Escobar, 1990). El objetivo principal es establecer la relación entre la estrategia metacognitiva para la compresión de texto bilingüe quechua-castellano en los estudiantes de la muestra.

La hipótesis planteada fue que, sí existe relación significativa entre la estrategia metacognitiva y la comprensión lectora con texto bilingüe quechua-castellano, en estudiantes de la serie 200 de la EFP de Enfermería de UNSCH-2019; de modo que, en el contexto de nuestra investigación, el aprendizaje de una segunda lengua permite desarrollar mayor activación cognitiva $y$, consecuentemente, mejor comprensión lectora. El diseño metodológico aplicado ha consistido en una investigación no experimental de nivel descriptivo correlacional causal; además, las estrategias utilizadas fueron la descripción, análisis y explicación de información de las variables en estudio.

Para lograr los resultados se utilizaron instrumentos como cuestionario estructurado y examen de compresión para fijar el nivel de desarrollo de la compresión lectora de los estudiantes bilingües. Aplicado los instrumentos pertinentes, se comprobaron que, sí existe correlación directa positiva entre los niveles de bilingüismo y la compresión lectora con textos bilingües.

Palabras clave: estrategias metacognitivas, la comprensión lectora y texto bilingüe

\section{ABSTRACT}

The research was aimed to explain the relationship between levels metacognitive strategies and reading comprehension in students from the 200 
series EFP Nursing UNSCH, 2019. The sociolinguistic context of students is mainly Castilian-Quechua bilingual. Most participants in the sample are placed according to the degree of mastery of languages in the different levels of bilingualism including: incipient, subordinating and coordinated. The main objective is to establish the relationship between metacognitive strategy text compression Quechua-Castilian bilingual students in the sample.

The hypothesis is that there exists significant relationship between levels metacognitive strategy and reading comprehension in bilingual text QuechuaCastilian, in students from the 200 series of VET Nursing UNSCH 2019, since in the context of our research, learning a second language allows humans to develop higher cognitive activation and consequently better reading comprehension, methodological design applied experimental research was descriptive not correlate causal level; In addition, the strategies used are description, analysis and explanation of information variables under study.

For the results it has been used instruments such as structured questionnaire and compression test to determine the development of reading comprehension level of bilingual students. Applied the relevant instruments, it has been concluded that if there is direct correlation between positive bilingualism levels and reading comprehension in bilingual text.

Keywords: Metacognitive strategies, reading comprehension and bilingual text

\section{INTRODUCCIÓN}

La comprensión lectora, en el fortalecimiento de la formación académica de los estudiantes universitarios, queda establecida como uno de los ejes fundamentales para la asimilación del conocimiento en el proceso formativo. Muchos estudiosos consideran que la lectura, como un acto complejo, facilita si se cuenta con una información previa acerca de su naturaleza, fines y características que poseen los textos y de los valores que en ellos se expresan; por último, acrecienta el conocimiento y es posible con el uso de algunas estrategias.

Los estudiantes de la educación básica regular y superior utilizan textos para comprender y, para esta actividad, deben aplicar estrategias metacognitivas que les permita efectivizar sus aprendizajes. Estas pericias involucran técnicas relacionadas con la cognición y la metacognición, las que consideramos un pareo inseparable en este trabajo de aprendizaje a partir de los textos científicos. Existen una diversidad de textos y, entre ellos, tenemos: narrativos, expositivos, descriptivos, etc. Tipos de texto considerados los más utilizados por los estudiantes universitarios; entonces, tenemos la intención de que sepan leer aplicando estrategias cognitivas y metacognitivas para que sinteticen sus aprendizajes a través de ellas. Dichas estrategias involucran procesos relacionados con la cognición y la metacognición.

Considerando las opiniones de los estudiosos en este campo, como Campanario (1994), Browny (1986), Baker (1994), Martí (1995), Palinsar y Brown (1997), entre otros, han estudiado las falencias de los estudiantes en la comprensión y retención de la lectura en sus diferentes niveles. Los resultados de estas averiguaciones son los que nos interesaron en nuestro trabajo de investigación. Para Flavell (1996), la función principal de una estrategia cognitiva es ayudar a alcanzar la metacognición 
aplicando las estrategias metacognitivas que tienen la función de establecer en qué nivel está progresando el estudiante referido a la compresión del texto. De tal modo que, la cognición ayuda a desarrollar el progreso cognitivo y la metacognición ayuda a controlar. Nos ha interesado saber cómo se dan algunos procesos metacognitivos al trabajar con textos narrativos bilingües (quechua-castellano) para posibilitar la comprensión del texto. Entender es la capacidad de identificar la temática variada que estimula el pensamiento, como: explicar, demostrar, ejemplificar, generalizar, volver a presentar el tópico de otra manera.

En la presente investigación se han utilizado textos en versión bilingüe, entendiendo que el bilingüe tiene un conocimiento más equilibrado de dos lenguas; mientras que el individuo que aprende una segunda lengua presenta un mayor dominio de la L1 que de la L2. La lengua siempre se usa dentro de un determinado contexto cultural, que varía de una lengua a otra. Si embargo, no quiere decir que una persona que conozca dos o más lenguas sea bicultural o multicultural.

Las estrategias de metacognición para la comprensión lectora con textos bilingües (quechua-castellano) dirigidas a los estudiantes de la serie 200 de la EFP de Enfermería-UNSCH Ayacucho, 2019, han sido el resultado de la preocupación inquietante de esclarecer las dificultades académicas de los estudiantes bilingües. Es constante escuchar que ser bilingües implica un desarrollo cognitivo deficiente; por tal razón, hemos planteado como objetivo: determinar las estrategias de metacognición para la comprensión lectora con textos bilingües (quechua-castellano) para los estudiantes bilingües. Se ha comprobado y sostenemos que las estrategias de metacognición para la compresión lectora con textos bilingües (quechua-castellano) tienen una correlación positiva considerable, con igual promedio de logros.

\section{MATERIALES Y MÉTODO}

\section{MÉTODO DE INVESTIGACION}

De acuerdo al objetivo general de la investigación se ha aplicado el método mixto, con mayor énfasis el descriptivo y analítico, porque tienen por objetivo identificar, clasificar, delimitar las variables de la investigación, porque requiere interpretar lo que es, es decir, está relacionada a condiciones o conexiones existentes, efectos que se sienten 0 tendencias que se relacionan. Correlacional porque ha buscado identificar probables relaciones de influencia entre variables con la finalidad de observar la dirección o grado de relación (Hernández, 2006)

\section{DISEÑO DE INVESTIGACIÓN}

Diseño no experimental, de dos variables de estudio.

TIPO DE INVESTIGACIÓN: Descriptivo correlacional

Descriptivo porque nos ha permitido describir la correlación de dos variables. Pues ha permitido utilizar las leyes de la pedagogía para mejorar los niveles de comprensión lectora que contribuyeron a lograr la competencia profesional en los estudiantes de la Escuela de Formación Profesional de Enfermería.

Carrasco (2005) refiere que se investiga para actuar, transformar, modificar o producir cambios en un determinado sector de la realidad. Villegas (2005) menciona que, es sin duda, el tipo de investigación más adecuado y necesario, en las actuales circunstancias, para la tarea educativa, porque el quehacer del maestro deber ser una permanente búsqueda de nuevas tecnologías y la adaptación y aplicación de nuevas teorías a la práctica de la educación, a la pedagogía experimental, con la finalidad de transformar la realidad educativa. 


\section{NIVEL DE INVESTIGACIÓN}

Básico que ha permitido describir la correlación entre las estrategias metacognitivas y la comprensión lectora en los estudiantes de la EFP de Enfermería UNSCH.

Valderrama (2003) señala que este nivel de investigación está dirigido a responder a la relación de los eventos físicos o sociales, su interés se centra en el por qué ocurre un fenómeno y en qué condiciones se da este.

Hernández et al. (2003) señalan que el nivel descriptivo responde a las relaciones de dos variables, sucesos y fenómenos físicos o sociales, cuyo objetivo principal es verificar la hipótesis correlacional, descubrimiento de nuevas leyes científicosociales.

\section{INSTRUMENTOS}

a) Cuestionario. Mediante este instrumento se ha obtenido una información valiosa de las estrategias metacognitivas. Se ha usado el cuestionario de respuesta indirecta.

Carrasco (2005) indica que el cuestionario consiste en presentar un documento, previa orientación y charla motivadora a los estudiantes. El conteniendo constará de una serie de preguntas ordenadas y coherentes, con claridad, precisión y objetividad, para que sean resueltas inmediatamente.

c) Examen. Para medir los niveles de comprensión lectora, se ha presentado textos de carácter académico con preguntas del nivel crítico.

\section{TÉCNICAS DE RECOLECCIÓN DE DATOS}

Guía de encuesta. Técnica que ha permitido entrar en contacto directo con los estudiantes de la muestra para el recojo de la información deseada.

Carrasco (2005) menciona que la encuesta es una técnica muy útil, versátil, sencilla y objetiva con la que se logra obtener los datos. Actualmente es muy usada en la investigación educacional, gracias a los resultados obtenidos por su aplicación.

Pruebas escritas. Ha permitido conocer los niveles de comprensión lectora que desarrollaron los estudiantes bilingües.

\section{DISCUSIÓN DE LOS RESULTADOS}

De los resultados obtenidos en la investigación, con el objetivo general planteado de establecer la relación que existe entre las estrategias metacognitivas y la compresión lectora en los estudiantes y en base a las calificaciones entre las estrategias metacognitivas y la compresión lectora, expresadas en escala vigesimal, con un $95 \%$ de confianza y $5 \%$ de significancia denotada, se ha interpretado la existencia de una correlación significativa. Los resultados de las correlaciones entre las estrategias metacognitivas y la comprensión lectora, han indicado que existe una relación positiva entre las calificaciones obtenidas en las estrategias metacognitivas que fueron directamente proporcionales a las calificaciones obtenidas en la comprensión lectora, y asociando a los promedios y desviaciones descritas en los párrafos anteriores, se ha determinado que existe una estabilidad en las calificaciones. Los resultados presentados hasta el momento, convertidos a escala cualitativa, han permitido rescatar algunos aspectos adicionales 
complementarios para describir mejor el comportamiento de la aplicación de estrategias metacognitivas y la comprensión lectora.

De los resultados ilustrados, entre las variables de estrategias metacognitivas y la comprensión literal, se pudo determinar que la comprensión lectora en el nivel literal, existe un porcentaje considerable de estudiantes con notas aprobatorias; es decir, a mejor aplicación de las estrategias metacognitivas es mejor el nivel de compresión lectora, y a menor aplicación de las estrategias metacognitivas, es menor el nivel de compresión lectora en textos bilingües (quechua y castellano). En términos generales, el resultado promedio ha sido "bueno", puesto que la condición de ser bilingüe ha permitido desarrollar mayores habilidades cognitivas, por tanto, la percepción de la realidad fue más holística, por lo que coincidimos con la investigación de Ochoa et al. (2005) en Colombia, quienes han demostrado a partir del abordaje de textos científicos que los estudiantes más regulares en el nivel educativo universitario tienen mejor control y monitoreo de sus procesos mentales. De la misma manera, se ha comprobado una directa correlación entre el funcionamiento metacognitivo y el nivel de comprensión: a mayor nivel de funcionamiento metacognitivo, mayor nivel de comprensión lectora y a menor nivel de funcionamiento metacognitivo menor nivel de comprensión lectora. Entonces, aplicando las estrategias metacognitivas para la compresión lectora en el nivel literal, sí se logra resultados positivos.

Los resultados entre las variables: estrategias metacognitivas y la comprensión inferencial se ha conseguido determinar que para la comprensión lectora en el nivel inferencial existe un porcentaje considerable con calificaciones entre "Deficiente y Regular" con un texto monolingüe (castellano); del mismo modo, las calificaciones de "Bueno y Muy Bueno" es mayor el porcentaje para el nivel de compresión inferencial con textos bilingües (quechua y castellano). En términos generales, el resultado promedio se ha ubicado en la estimación de "Regular a Bueno", debido a que la condición de ser bilingüe ha permitido desarrollar mayores habilidades cognitivas y una percepción global de la realidad. En consecuencia, coincidimos con la investigación de Herrera (2005), realizada con dos grupos de estudiantes del Instituto Alberto Merani, Colombia, que tuvo como propósito identificar la relación existente entre las estrategias metacognitivas y la comprensión lectora, se encontró aquellos estudiantes que recibieron entrenamiento en estrategias metacognitivas mostraron un significativo avance en los procesos de comprensión lectora.

Con los resultados de las estrategias metacognitivas y la comprensión crítica, hemos determinado que, en la comprensión lectora en el nivel crítico, las calificaciones el $48 \%$ se han ubicado entre "Deficiente y Regular". Por otro lado, han logrado comprender el texto bilingüe el $52 \%$ que se ubicaron en la estimación de "Bueno y Muy Bueno", por lo que hemos deducido que falta un mayor afianzamiento en el uso de las estrategias metacognitivas para el mejor logro de la compresión lectora con textos bilingües (quechua y castellano).

Con los resultados obtenidos en la investigación, hemos determinado que las estrategias metacognitivas tienen ventajas favorables para la compresión de textos bilingües (quechua y castellano), ayuda al estudiante a mejorar su desenvolvimiento social, académico y, con mayor énfasis, en la compresión 
lectora. Además, la comunicación humana es importante en toda su dimensión, para llegar a construir un país inclusivo en la educación, como un derecho. En Ayacucho tenemos mayor porcentaje de estudiantes provenientes de la zona rural, por ende, son bilingües y requieren profesionales formados en Educación Intercultural Bilingüe. Además, la creación de nuevas instituciones es una necesidad, especialmente en las zonas rurales y urbanomarginales para que puedan acceder a una educación de calidad; por tanto, se debe formar profesores bilingües, que desarrollen la competencia comunicativa de los estudiantes bilingües.

\section{RECOMENDACIONES}

Se debe revalorar la lengua originaria de los estudiantes y usarla en todos los procesos de formación profesional, superando los perjuicios lingüísticos hasta ahora observados y contribuir al desarrollo de la compresión lectora.

Es necesario que los profesores dominen las estrategias metacognitivas de la comprensión lectora en sus diferentes niveles: literal, inferencial y crítica, durante el proceso de la enseñanza-aprendizaje.

Cumplir la Ordenanza Regional, que reconoce el carácter multilingüe de la región ayacuchana, tanto en el ámbito rural y urbano, con efecto práctico de la diversidad cultural y de idiomas como instrumento de comunicación social, cultural y educativa en castellano, quechua, asháninca y machiguenga, lenguas oficiales.

\section{REFERENCIAS BIBLIOGRÁFICAS}

Allende, f. Condemarín (1986) La lectura: teoría, evaluación y desarrollo. Andrés Bello. Santiago.

Aragón, E. y Caicedo, A. (2009). La enseñanza de estrategias metacognitivas para el mejoramiento de la comprensión lectora. Estado de la cuestión. Pensamiento Psicológico, Vol. 5, Núm. 12, enero-junio, pp. 125-138. Pontificia Universidad Javeriana. Colombia. Material en la Red. Consulta realizada en julio de 2011.http://redalyc.uaemex.mx/src/inicio/ArtPdfRed.jsp?iCve=80111899010.

Areiza y Henao, L. (1999), Metacognición y estrategias lectoras. Revista de Ciencias Humanas, 19. Material en la Red. Consulta realizada en mayo de 2009. Disponible en:http://www.utp.edu.co/ chumanas/revistas/revistas/rev19.areiza.htm

Baker, L. y Brown, A. (1984) "Cognitivemonitoring in Reading" en Flood, J. (Eds.) Understanding Reading Comprehension: Cognition, Language and the Structure of Prose. Delaware: I.R.A.

Barnett, M. (1989) La lectura del idioma extranjero y práctico. New Jersey.

Barthes, R. (1987) Sobre la lectura. El susurro de allá de la palabra y la escritura. Barcelona: Paidós. 
Bloomfield, L. (1964) Lenguaje. Universidad Mayor de San Marcos, Lima-Perú.

Bracho, F. (2008) Correlación entre la evolución de la lectura oral y los niveles de comprensión lectora del discurso académico en estudiantes de educación. Trabajo para optar al título de Magister en la enseñanza de la lectura y la escritura. Santa Ana de Coro: Universidad Nacional Experimental "Francisco de Miranda".

Bravo, V. (2008) Evolución de los niveles de comprensión lectora del discurso académico en los estudiantes de Educación Mención Lengua, Literatura. Trabajo para optar al título de Magister en la Enseñanza de la Lectura y la Escritura. Santa Ana de Coro: Universidad Nacional Experimental "Francisco de Miranda".

Brown, K. (2006) Encyclopedia of language \& linguistics. Oxford: Elsevier.

Campanario, J. y otros (1998). Metacognición y el aprendizaje de las ciencias. Universidad de Rioja España. Investigación e innovación en la enseñanza de las ciencias. Material en la Red. Consulta realizada en mayo de 2010. Disponible en: http://dialnet.unirioja.es/servlet/articulo?codigo $=993584$

Carrasco, S. (2005) Metodología de la Investigación Científica: pautas metodológicas para diseñar y elaborar proyectos de investigación. Ed. San Marcos. Lima

Cassany, D. (2004) Revista Latinoamericana de Lectura. Lectura y Vida. Año 25. No. 2. Junio. (p.p. 6-23) ------------. (2004) El Dictado como Tarea Comunicativa, Universitat Pompen Fabra.

Cassany, D. y otros (1990) Cómo aprender a leer. GRAO. Barcelona.

Cerchiaro, E. y otros (2010) Un acercamiento a la metacognición y la comprensión lectora en estudiantes universitarios de México y Colombia. Universidad del Magdalena. Santa Marta.

Cerron-Palomino, R. (1976) Lingüística Quechua. CERA-BC. Cusco.

Cummins, J. (1984) La influencia de bilingüismo en el desarrollo cognitivo: una síntesis de los resultados de la investigación y las hipótesis explicativas.

Da Costa, F. (2008) Uso de la nominalización en textos académicos universitarios y niveles de comprensión alcanzados por los estudiantes al leer los textos referidos. Trabajo Especial de grado para optar al título de Magister Scientiarum en la Enseñanza de la Lectura y la Escritura. Santa Ana de Coro: Universidad Nacional Experimental "Francisco de Miranda".

Degregori, C. (2001) Perú: Identidad, nación y diversidad cultural en Interculturalidad (creación de un concepto y desarrollo de una actitud) Programa FORTE-PE/PROEBI Editor: María IESE; Lima.

Dubois, M. (1991) El proceso de la lectura, enseñar a comprender: Aique. Buenos Aires.

Escobar, A. M. (1990) Los bilingües y el castellano en el Perú. Instituto de estudios peruanos. Lima- Perú.

Ferguson, Ch. (1975) Antropología Lingüística, BLITZ, España. 
Ferreiro, E. (2001). Pasado y presente de los verbos leer y escribir. Fondo de Cultura Económica: México. 95 p.p.

González, F. (1996). Acerca de la Metacognición. Revista Paradigma, Vols. XIV al XVII, 1993-1996; 109-135.

González, R. y otros (2004) Desarrollo de competencias lectoras y escritoras en estudiantes de Ciclo Nivelatorio, General y de Facultad de la Universidad del Magdalena. Grupo de Investigación Institucional de Lenguaje. Universidad del Magdalena. Santa Marta.

Grosjean, F. (1992) Bilingüe La persona del bicultural en el oído en el Mundo sordo. El Idioma de la señal Estudia, v. 77, pág. 307-320

Hernández, R. (2006) Metodología de la Investigación. México. Edit. McGraw Hill Interamerican S.A.

Herrera y otros (2011) Habilidades metacognitivas en estudiantes de una universidad pública y una privada de Mérida. Universidad Marista, Universidad Autónoma de Yucatán. En Revista Científica Electrónica de Psicología. ICSa-UAEH. №11 ISSN 1870-5812 Material en la Red Consulta realizada en octubre de 2011.

Disponible en http://dgsa.uaeh.edu.mx/revista/psicologia//MG/pdf/No. 19 - 11.pdf

Herrera, J. (2009) Metacognición en estudiantes de una universidad pública y una universidad privada. Tesis de pregrado no publicada, Universidad Marista de Mérida, México.

Herrera, y otros (2004). Una forma de procesar la información en los textos científicos y su influencia en la comprensión. Revista Electrónica de Investigación Educativa. México. Material en la Red. Recuperado en agosto de 2011. Disponible en: redalyc.uaemex.mx/pdf/155/15506101.pdf

Johnston, (1989) Evaluación de la comprensión lectora. Un enfoque cognitivo. Visor, Madrid

Lambert (1974) La cultura e Idioma como los factores aprendizaje en la educación. los Factores de la Cultura Aprendiendo. Bellingham: Washington occidental la Universidad Estatal.

Mackey, W. F. y Siguán, M. (1986) Educación y bilingüismo. Madrid: Santillana/UNESCO.

Massone, M. y MACHADO, M. Lengua de señas argentina: análisis de vocabulario bilingüe. Buenos Aires: Edicial, 1994.

Mateos, M. (1995). Programas de intervención metacognitiva dirigidos a la mejora de la comprensión lectora. Características y Efectividad. En comp. de Carretero, M; Almaraz, J. Y Fernández B., P. Razonamiento y comprensión. Madrid, Trotta. 